\title{
A 30-GHz Self-Injection-Locked Oscillator Having a Long Optical Delay Line for Phase-Noise Reduction
}

\author{
Kwang-Hyun Lee, Jae-Young Kim, and Woo-Young Choi
}

\begin{abstract}
We demonstrate a millimeter-wave self-injectionlocked (SIL) oscillator having a long optical delay line as a feedback route. In the SIL oscillator, a part of output signal is self-injected into the oscillator after passing through a long optical delay line, resulting in locked oscillation and phase-noise reduction. By controlling the self-injection power, we achieve 30-GHz oscillation with a sidemode suppression ratio larger than $50 \mathrm{~dB}$ and about 18-dB phase-noise reduction at $10-\mathrm{kHz}$ frequency offset.
\end{abstract}

Index Terms-Optoelectronic oscillator (OEO), self-injection locking, single-mode oscillation.

\section{INTRODUCTION}

$\mathbf{O}$ SILLATORS are one of the key components required in many communication systems for commercial and military applications, and their stability and low phase-noise characteristics are important performance parameters.

A self-injection-locked (SIL) oscillator has been actively investigated for generation of stable and low phase-noise signals. It can be easily realized by self-injection of a part of output signals after passing through a high quality $(Q)$-factor external resonator or a long delay line. Many successful demonstrations have been reported [1]-[4]. For example, phase noises of about $-50 \mathrm{dBc} / \mathrm{Hz}(20-\mathrm{dB}$ phase-noise reduction) at $10-\mathrm{kHz}$ offset for 8-GHz band [1] and $-120.1 \mathrm{dBc} / \mathrm{Hz}$ (4-dB phase-noise reduction) at 1-MHz offset for 9.6-GHz band [3] have been achieved by a delay line in the feedback loop, while $-95 \mathrm{dBc} / \mathrm{Hz}$ at $100-\mathrm{kHz}$ offset for $60-\mathrm{GHz}$ band has been obtained by a ceramic high- $Q$ resonator in the loop [4].

Although it is possible to further reduce phase noises in the $\mathrm{RF}$ domain by using a longer delay line or a higher $Q$ external resonator, they are very impractical especially for millimeterwave applications, since the delay line length is limited by large loss and high- $Q$ devices are not easily available [5].

However, long optical delay lines have been used for generation of a very high- $Q$ external resonator in an optoelectronic oscillator (OEO) because of low transmission loss of optical fiber [6], [7]. An OEO can generate high-spectral-purity micro/millimeter waves with a long optical delay line, but the optical loop should have loop gain for self-oscillation requiring very

Manuscript received June 21, 2007; revised August 20, 2007.

The authors are with the Department of Electrical and Electronic Engineering, Yonsei University, Seoul 120-749, Korea (e-mail: wchoi@yonsei.ac.kr).

Color versions of one or more of the figures in this letter are available online at http://ieeexplore.ieee.org.

Digital Object Identifier 10.1109/LPT.2007.909684

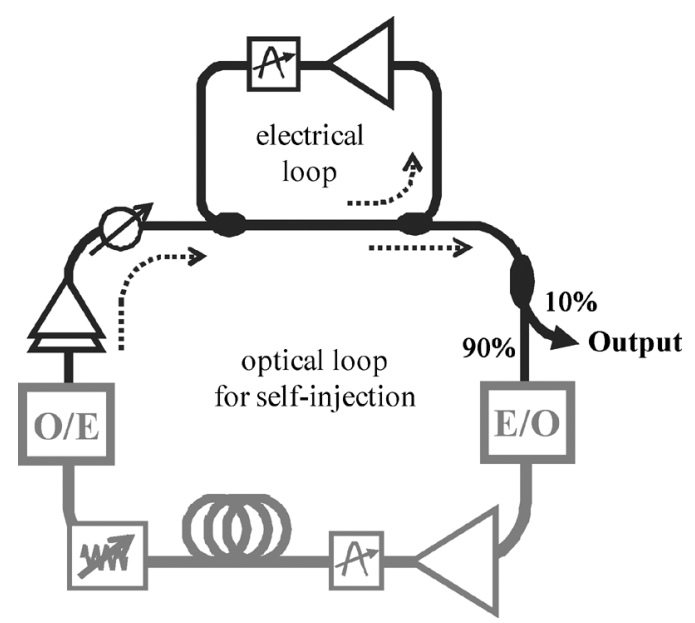

Fig. 1. Configurations of the proposed SIL oscillator having a long optical delay line.

high electrical and optical gain to compensate the large electrical-to-optical-to-electrical (E/O/E) conversion loss.

In this letter, we demonstrate an SIL oscillator with a long optical delay line that can significantly reduce the output phase noise. Its configuration is shown in Fig. 1. Unlike OEOs, the optical loop works as a passive feedback loop. With this SIL oscillator, we successfully demonstrate single-mode oscillation at 30-GHz bands with a sidemode suppression ratio (SMSR) larger than $50 \mathrm{~dB}$ and output phase-noise reduction of about $18 \mathrm{~dB}$ at $10-\mathrm{kHz}$ frequency offset. In addition, dependence of SMSR and the output phase noise on the self-injection power is investigated.

\section{Theory AND Operating PRINCIPLE}

Our SIL oscillator is composed of a hybrid of an electrical and an optical loop. The electrical loop has sufficient gain to oscillate by itself, while the optical loop does not. Therefore, the optical loop works as a feedback route for self-injection of electrical signals.

A part of output signals from the electrical oscillator, as can be seen in Fig. 1, is injected into the oscillator after passing through a long optical delay line and it locks the electrical oscillator, achieving self-injection locking. Once the oscillator is locked by the delayed replica of its output signal, its frequency and phase fluctuations are reduced and the phase-noise reduction ratio $(\eta)$ near the carrier frequency can be described as [1], [2]

$$
\lim _{\omega \rightarrow 0} \eta(\omega) \rightarrow \frac{1}{\left(1+\sqrt{\kappa} \omega_{3 \mathrm{~dB}} \tau\right)^{2}}
$$


where $\omega$ is the offset frequency from the center frequency, $\tau$ is the optical-loop delay, $\omega_{3} \mathrm{~dB}$ is the half-width at half-maximum of the resonator inserted in the electrical oscillator, and $\kappa$ is the self-injection power normalized to the oscillator output power. In (1), it is assumed that there is no steady-state phase difference between self-injected and oscillator output signals. As shown in (1), a longer delay line or larger power injection produces more phase noise reduction. In our experiment, 2.4-km-long singlemode fiber (SMF) was used as a delay line.

\section{EXPERIMENTAL SETUP AND RESULTS}

As shown in Fig. 1, the electrical loop was configured with an electrical amplifier having RF gain of about $18 \mathrm{~dB}$, an RF filter having $Q$ of 1000 at $30 \mathrm{GHz}$ with about 3.16-dB insertion loss, and a four-port 3-dB RF coupler having insertion loss of about $1.8 \mathrm{~dB}$. Ninety percent of output signals from the electrical loop were converted to the optical signal by an E/O converter made up of a tunable laser source and a Mach-Zehnder modulator (MZM) having 40-GHz modulation bandwidth, and passed through 2.4-km-long SMF.

Optical signals were converted to electrical signals by a highspeed photodiode having $60-\mathrm{GHz}$ bandwidth and self-injected to lock the electrical oscillator. An Er-doped fiber amplifier and two electrical amplifiers having the total gain of about $46 \mathrm{~dB}$ were inserted to partially compensate large conversion $(\mathrm{E} / \mathrm{O} / \mathrm{E})$ loss, and an optical filter was inserted for filtering out the lower sideband in MZM output signals to avoid any RF signal-fading problem induced by fiber dispersion [8]. The power and phase of self-injected signals were adjusted by an optical attenuator and an RF phase shifter, respectively.

Ten percent of output signals from the electrical loop were measured by an RF spectrum analyzer (HP8563E) connected with an external mixer (HP11970A) having conversion loss of about $26 \mathrm{~dB}$ after attenuation of about $2.67 \mathrm{~dB}$. This attenuation was needed due to the display limit $(-6.1 \mathrm{dBm})$ of the spectrum analyzer used in the measurement.

The output spectra measured at various $\kappa$ are shown in Fig. 2. Fig. 2(a) is the output spectrum measured at $\kappa=0$ (no self-injection), while (b) and (c) are the spectra measured at $\kappa$ of $1.6 \times 10^{-3}$ and $2.5 \times 10^{-1}$, respectively. These figures show that self-injection-locking reduces phase noise and larger $\kappa$ provides larger improvement. However, unwanted sidemodes separated by about $84 \mathrm{kHz}$ from the center frequency can be observed in Fig. 2(b) and (c), and they increase as $\kappa$ increases. These are due to coupled-loop oscillation. Although the optical loop cannot oscillate alone due to large loop loss, the coupled-loop can oscillate if electrical-loop gain compensates optical-loop loss [9]. Therefore, as the injection power increases, causing optical-loop loss reduction, the coupled-mode becomes stronger. These unwanted signals can be suppressed by adding an additional optical delay line having different length [10].

In order to clearly verify phase-noise reduction performance, single-sideband (SSB) phase noises of output signals shown in Fig. 2 were measured and the results are shown in Fig. 3. The measured phase-noise values at $10-\mathrm{kHz}$ frequency offset are $-91.83 \mathrm{dBc} / \mathrm{Hz}(\kappa=0),-110.17 \mathrm{dBc} / \mathrm{Hz}\left(\kappa=1.6 \times 10^{-3}\right)$, and $-118.5 \mathrm{dBc} / \mathrm{Hz}\left(\kappa=2.5 \times 10^{-1}\right)$. The phase-noise peaking

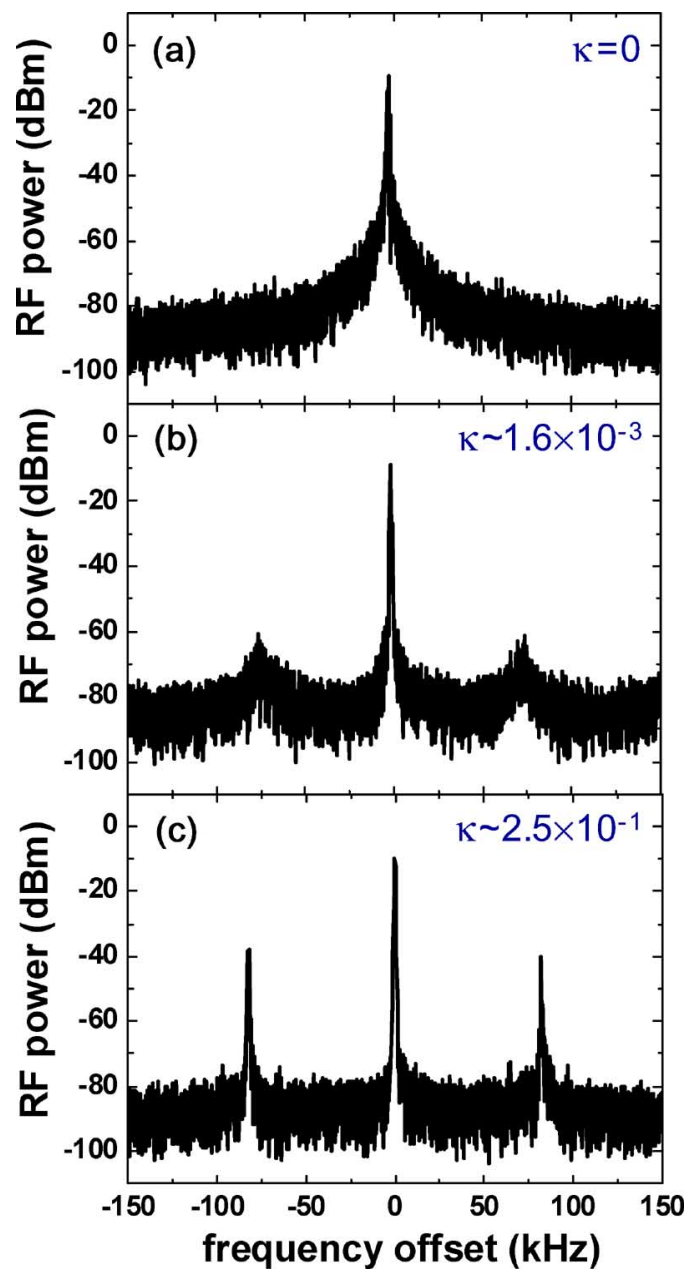

Fig. 2. Measured RF spectra of the output signals at (a) $\kappa=0$, (b) $\kappa \sim$ $1.6 \times 10^{-3}$, and (c) $\kappa \sim 2.5 \times 10^{-1}$. The center frequencies are 29.9773362 , 29.9770507 , and $29.9768307 \mathrm{GHz}$, respectively. In all figures, the frequency span and resolution bandwidth settings were $300 \mathrm{kHz}$ and $300 \mathrm{~Hz}$, respectively.

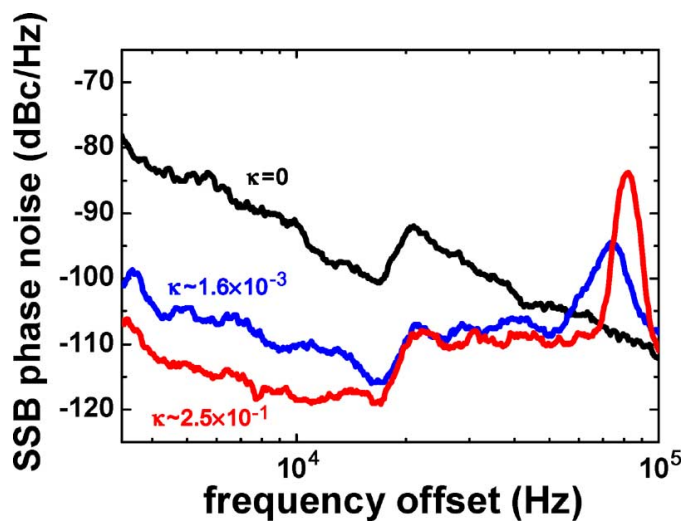

Fig. 3. Measured phase noises of the output signals at $\kappa=0, \kappa \sim 1.6 \times 10^{-3}$, and $\kappa \sim 2.5 \times 10^{-1}$.

at $\sim 20-\mathrm{kHz}$ frequency offset is believed due to our measurement setup using an external mixer. Clearly, the self-injection locking drastically enhances phase quality of output signals.

Fig. 4 shows dependency of SSB phase noises measured at $10-\mathrm{kHz}$ frequency offset and SMSR on $\kappa$. As shown in the figure, both SMSR and SSB phase noises are reduced with 


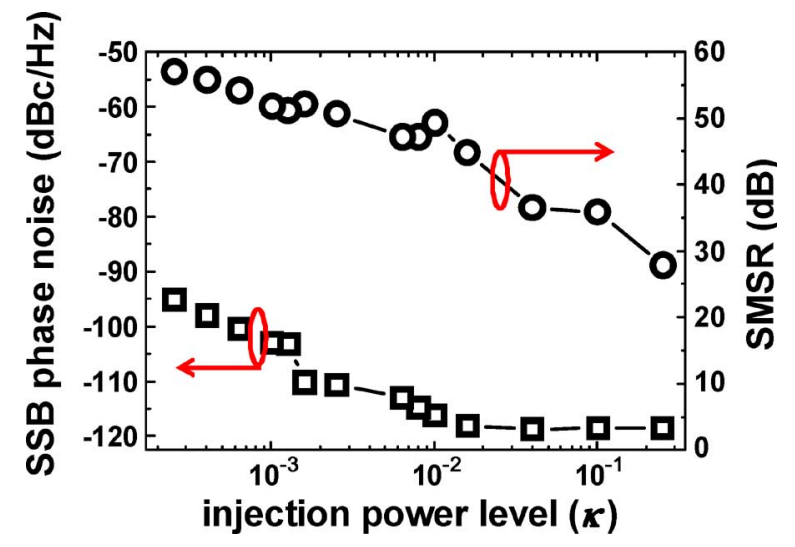

Fig. 4. SSB phase noise at $10-\mathrm{kHz}$ frequency offset from the center frequency and SMSR according to the injection power level.

increasing $\kappa$. For $\kappa$ larger than $10^{-2}$, phase noises are saturated due to the sensitivity limit of our spectrum analyzer used in the measurement.

\section{CONCLUSION}

We have demonstrated SIL oscillators with 2.4-km-long SMF as a long feedback line for output phase-noise reduction at 30-GHz bands and found that the higher self-injection power provides larger phase-noise reduction but with lower SMSR.
At the injection-power level of about $1.6 \times 10^{-3}, 30-\mathrm{GHz}$ oscillation with SMSR larger than $50 \mathrm{~dB}$ and output phase-noise reduction of about $18 \mathrm{~dB}$ at $10-\mathrm{kHz}$ frequency offset have been successfully demonstrated.

\section{REFERENCES}

[1] H.-C. Chang, "Phase noise in self-injection-locked oscillators," IEEE Trans. Microw. Theory Tech., vol. 51, no. 9, pp. 1994-1999, Sep. 2003.

[2] H.-C. Chang, "Stability analysis of self-injection-locked oscillators," IEEE Trans. Microw. Theory Tech., vol. 51, no. 9, pp. 1989-1993, Sep. 2003.

[3] T.-P. Wang, Z.-M. Tsai, K.-J. Sun, and H. Wang, "Phase-noise reduction of X-band push-push oscillator with second-harmonic self-injection techniques," IEEE Trans. Microw. Theory Tech., vol. 55, no. 1, pp. 66-77, Jan. 2007.

[4] F. Kuroki, S. Ishikawa, and T. Yoneyama, "Comparative study on two types of self-injection locked NRD guide gunn oscillators," in Proc. Eur. Microw. Conf., Paris, France, 2005.

[5] M. Kaba, H.-W. Li, A. S. Daryoush, J.-P. Vilcot, D. Decoster, J. Chazelas, G. Bouwmans, Y. Quiquempois, and F. Deborgies, "Improving thermal stability of opto-electronic oscillators," IEEE Microw. Mag., vol. 7, no. 4, pp. 38-47, Aug. 2006.

[6] X. S. Yao and L. Maleki, "Optoelectronic microwave oscillator," J. Opt. Soc. Amer. B., vol. 13, no. 8, pp. 1725-1735, Aug. 1996.

[7] X. S. Yao and L. Maleki, "Converting light into spectrally pure microwave oscillation," Opt. Lett., vol. 21, no. 7, pp. 483-485, Apr. 1996.

[8] U. Gliese, S. Norskov, and T. N. Nielsen, "Chromatic dispersion in fiber-optic microwave and millimeter-wave links," IEEE Trans. Microw. Teory Tech., vol. 44, no. 10, pp. 1716-1724, Oct. 1996.

[9] D. Eliyahu and L. Maleki, "Low phase noise and spurious level in multi-loop optoelectronic oscillators," in Proc. IEEE Int. Frequency Control Symp., 2003, pp. 405-410.

[10] X. S. Yao and L. Maleki, "Multiloop optoelectronic oscillator," IEEE J. Quantum Electron., vol. 36, no. 1, pp. 79-84, Jan. 2000. 